\title{
ESTUDIO NUMÉRICO DEL EFECTO DE LAS VARIABLES DE CONTROL EN LA AUTOIGNICIÓN DEL GAS DE SÍNTESIS EN UN MOTOR HCCI ESTACIONARIO PARA MICROGENERACIÓN
}

\section{NUMERICAL STUDY OF THE EFFECT OF THE CONTROL VARIABLES ON THE AUTOIGNITION OF SYNTHESIS GAS IN A STATIONARY HCCI ENGINE FOR MICROGENERATION}

\author{
Ing. Andrés David Morales-Rojas*, PhD. Iván Darío Bedoya-Caro *** \\ *Institución Universitaria Pascual Bravo, Facultad de Ingeniería, Grupo de \\ Investigación e Innovación en Energía (GIIEN). \\ Calle 73 \# 73A - 226, Medellín, Antioquia, Colombia. \\ Teléfono: +57 (4) 4480520, E-mail: andres.morales@pascualbravo.edu.co. \\ ${ }^{* *}$ Universidad de Antioquia, Facultad de Ingeniería, Grupo del Gas y Uso Racional de la \\ Energía (GASURE). \\ Calle 67 \# 53 - 108, Medellín, Antioquia, Colombia. \\ Teléfono: +57 (4) 2198548, E-mail: Ivan.bedoya @udea.edu.co.
}

\begin{abstract}
Resumen: Los motores HCCI han surgido como respuesta a las exigentes normas de regulación de emisiones contaminantes y a la inminente escasez de combustibles fósiles gracias a que combinan una baja emisión de contaminantes y una alta eficiencia térmica. Dado que la combustión HCCI depende en gran medida de la cinética química de la mezcla combustible-aire admitida, la cual a su vez depende de la composición, temperatura, presión y concentración de la mezcla; una buena aproximación del orden de magnitud de estas variables es indispensable para hacer un ajuste preliminar del sistema de control. En este estudio, se analizan los efectos de la variación en la composición del gas de síntesis, el dosado relativo y las condiciones de temperatura y presión en la admisión como parámetros de control de la autoignición en un motor estacionario para microgeneración; a través de un análisis numérico utilizando un modelo cero dimensional de una zona con cinética química detallada, empleando el programa CHEMKIN-PRO®. Los resultados muestran que la variación de la temperatura juega el papel predominante en determinar el inicio de la combustión. Las demás variables también juegan un papel importante pero su efecto es menor.
\end{abstract}

Palabras clave: Gas de síntesis, motores HCCI, análisis numérico, cinética química, combustión avanzada.

\begin{abstract}
HCCI engines have emerged in response to the stringent regulations of polluting emissions and the imminent scarcity of fossil fuels thanks to the combination of low emission of pollutants and high thermal efficiency. Given that HCCI combustion depends to a large extent on the chemical kinetics of the admitted fuel-air mixture, which in turn depends on the composition, temperature, pressure and concentration of the mixture; a good approximation of the order of magnitude of these variables is essential to make a preliminary adjustment of the control system. In this study, the effects of the variation in the composition of the synthesis gas, the equivalence ratio and the conditions
\end{abstract}


of temperature and pressure in the admission are analysed as ignition control parameters in a stationary motor for microgeneration; through a numerical analysis using a one zone zero dimensional model with detailed chemical kinetics, using the software CHEMKINPRO®. Results show that temperature variation plays the predominant role in determining the start of combustion. The other variables also play an important role but their effect is less

Keywords: Synthetic gas, HCCI engines, numerical analysis, chemical kinetics, advanced combustion.

\section{INTRODUCCIÓN}

En Colombia, las zonas no interconectadas a la red eléctrica (ZNI) suman un $51 \%$ del territorio nacional. En la actualidad, en estas zonas del país se dispone de una capacidad operativa de generación eléctrica de $241 \mathrm{MW}$, de la cual solamente un $3 \%$ corresponde a FNCER ${ }^{1}$ (Rey Luengas et al., 2018). El mayor porcentaje de energización de las ZNI se encuentra en las cabeceras departamentales y municipales, las cuales cuentan generalmente con generadores diesel y, en algunos casos, con pequeñas centrales hidroeléctricas y fotovoltaicas. Las ZNI se encuentran aisladas energéticamente del resto del territorio nacional debido a sus características geográficas y naturales, $\mathrm{y}$, por lo tanto, la prestación del servicio de energía eléctrica debe generarse en cada zona. En consecuencia, existe una necesidad de adquirir combustibles para la generación eléctrica local y para consumo doméstico. Sin embargo, las ZNI no cuentan con la infraestructura que permita el abastecimiento de energéticos a partir de los medios de transporte tradicionales (poliductos y gasoductos), lo cual conlleva a que el costo de los energéticos se eleve notoriamente y que el suministro no sea confiable (Esteve Gómez, 2011). En particular, en las ZNI existen necesidades de generación de potencia o energía eléctrica - de hasta $50 \mathrm{~kW}$ - en poblados pequeños que no cuentan con la cobertura de los centros poblados más grandes pero que cuentan con acceso a grandes cantidades de biomasa residual producto de actividades agropecuarias, lo que posibilita la aplicación de tecnologías para su transformación y aprovechamiento energético.

La biomasa es considerada una de las fuentes energéticas históricamente más aprovechadas por

${ }^{1}$ Fuentes no convencionales de energía renovables. la humanidad y en la actualidad participa con un $10 \%$ de la matriz energética mundial (Agency, 2018; McKendry, 2002a). La biomasa se destaca como una de las principales opciones de la llama transición energética gracias a su disponibilidad, poca dependencia de su producción con las condiciones ambientales y facilidad de conversión. Otra de las ventajas de utilizar biomasa es que el vertimiento de dióxido de carbono $\left(\mathrm{CO}_{2}\right)$ en la atmósfera es contrarrestado por la siembra de nuevos cultivos, por lo que cobra gran interés en la política mundial de descarbonización del sector energético y dado que, en algunos casos, la biomasa se considera un residuo, el costo asociado a su aprovechamiento es mucho más bajo que los obtenidos con otros tipos de energéticos.

El sector agropecuario colombiano genera gran cantidad de material orgánico de desecho como subproducto de su actividad de producción (Escalante et al, 2011). En la mayoría de los casos, este material es usado como abono en los cultivos o como combustible quemado en calderas y en hornos de secado. El problema, es que muchos procesos en el sector agropecuario dependen de la combustión de combustibles gaseosos para la obtención de calor o requieren energía para sistemas mecánicos o eléctricos, por lo que la combustión directa de biomasa no es una alternativa viable. Se debe tener en cuenta que la biomasa, es la única fuente de energía renovable que puede ser transformada en combustibles gaseosos, líquidos o sólidos, mediante procesos de conversión termoquímicos y bioquímicos (Escalante et al., 2011; McKendry, 2002a). Estos combustibles pueden ser empleados en procesos de generación de calor por combustión directa o en generación eléctrica por ciclos termodinámicos de potencia; el uso más común para los combustibles líquidos y gaseosos, obtenidos a partir de biomasa, es su implementación en motores de combustión 
interna alternativos (MCIA) y en turbinas de generación (Ciferno y Marano, 2002; McKendry, 2002a; Quaak, Harrie, y Stassen, 1999). (L Tangarife et al., 2017); (A Niño, 2018).

La gasificación es un proceso termoquímico de transformación de la biomasa en un combustible gaseoso, llamado gas de síntesis. En la gasificación, aire, oxigeno $\left(\mathrm{O}_{2}\right)$, vapor de agua $\left(\mathrm{H}_{2} \mathrm{O}\right)$ o una mezcla de ellos reacciona de manera parcial con la biomasa; las reacciones toman lugar a temperaturas entre los 500 y $1400^{\circ} \mathrm{C}$ y a condiciones de presión atmosférica o tan altas como 33 bar (Ciferno y Marano, 2002). Dependiendo de la tecnología, la biomasa, el agente gasificante y las condiciones de operación del sistema, el gas de síntesis estará formado por cantidades variables de hidrogeno $\left(\mathrm{H}_{2}\right)$, monóxido de carbono (CO), metano $\left(\mathrm{CH}_{4}\right)$, pequeñas cantidades de hidrocarburos pesados $(\mathrm{C}+)$, dióxido de carbono, vapor de agua $\left(\mathrm{H}_{2} \mathrm{O}\right)$ y nitrógeno $\left(\mathrm{N}_{2}\right)$ (Ciferno y Marano, 2002; McKendry, 2002c). Los gasificadores que utilizan aire como agente gasificante producen un gas, conocido como gas pobre, que tiene gran cantidad de $\mathrm{N}_{2}$ y bajo poder calorífico - 4 a $6 \mathrm{MJ} / \mathrm{Nm}^{3}-$, el cual puede ser utilizado en procesos de combustión directa o como combustible en MCIA (Ciferno y Marano, 2002; McKendry, 2002b, 2002c).

Los MCIA son considerados como una de las tecnologías de transformación energética con más aplicación en todo el mundo, especialmente cuando existen variaciones en los requerimientos de demanda energética y de potencia. Esta popularidad se debe principalmente a que los MCIA son una tecnología reconocida, económica y confiable, con posibilidad de uso en sistemas estacionarios para generación energética y de potencia, así como en sistemas automotrices. Tradicionalmente, estos motores utilizan gasolina, diesel y más recientemente gas natural (GN). Sin embargo, existe un gran interés en la aplicación de gran variedad de combustibles alternativos, especialmente los de origen renovable (Richards, Casleton, y Weiland, 2009). El gas de síntesis puede ser utilizado como combustible tanto en motores de encendido provocado (MEP), como en motores de encendido por compresión (MEC) convertidos a modo dual. Para que la aplicación tenga éxito técnico y económico, el gas debe contar con un poder calorífico lo suficientemente alto y, además, debe estar libre de impurezas y alquitranes, a fin de minimizar los problemas de mantenimiento (Quaak et al., 1999). En general, los diferentes estudios reportados en la literatura muestran una pérdida de potencia en motores que utilizan gas de síntesis en comparación con los que utilizan combustibles convencionales. Está perdida de potencia es atribuida al desplazamiento del aire ocasionado por la baja densidad del gas de síntesis más que a su bajo poder calorífico (Hagos et al, 2014); (J Plaza, M Núñez, 2017).

Dos de los principales retos para la industria de fabricación de MCIA son: 1- el desarrollo de tecnologías con alta eficiencia de transformación energética y 2- el cumplimiento de las cada vez más estrictas normas de regulación de emisiones de gases contaminantes y de efecto invernadero (Dec, 2009; "Emission Standards," n.d.; Heywood, 1988). Los motores de encendido provocado, con sus sistemas de tratamiento de gases, han sido exitosos en minimizar las emisiones de óxidos de nitrógeno (NOx), monóxido de carbono e hidrocarburos sin quemar (HC); mientras que, debido a las bajas relaciones de compresión y al uso de válvula mariposa, su eficiencia térmica es baja, especialmente en grados medios y bajos de carga. Por otro lado, los motores de encendido por compresión, ofrecen altas eficiencias térmicas debido a sus mayores relaciones de compresión y a la usencia de válvula mariposa y, gracias al uso de dosados relativos $(\varnothing)$ pobres, emiten bajas cantidades de dióxido de carbono por unidad de trabajo producido; aunque existen tecnologías de tratamiento de gases para MEC que ayudan a disminuir la emisión de $\mathrm{NOx}, \mathrm{CO}, \mathrm{HC}$ y material particulado (MP), su aplicación se ve limitada debido a su alto costo de adquisición y mantenimiento (Saxena y Bedoya, 2013); (O Suarez at el, 2018).

\subsection{Generalidades de los motores HCCI}

Una alternativa a las tecnologías de combustión tradicionales en MCIA es la combustión HCCI Homogeneous Charge Compression Ignition -, por sus siglas en ingles. La combustión HCCI ofrece una alta eficiencia térmica y bajas emisiones de óxidos de nitrógeno y de material particulado si se compara con las obtenidas en los motores de encendido provocado y los motores de encendido por compresión (Dec, 2009; Hasan y Rahman, 2016; Saxena y Bedoya, 2013; Yao et al, 2009); también, pueden ser adaptados para funcionar con diferentes tipos de combustibles, incluso los de origen renovable. En términos generales, un motor HCCI admite una mezcla combustible-aire, tal como ocurre en un MEP, pero a diferencia de este, la ignición no es inducida por una descarga eléctrica, sino que se da gracias a las características cinético-químicas de la mezcla admitida y al incremento de la temperatura de esta en la carrera 
de compresión, algo similar a lo ocurrido en un MEC. En vista de que se admite una mezcla combustible-aire, la ignición se da de manera casi homogénea en toda la cámara de combustión, generando así, tasas de liberación de energía y picos máximos de presión muy altos; por lo que, para evitar daños en los componentes estructurales del motor - culata, válvulas y pistón - el combustible admitido debe estar altamente diluido, ya sea por la operación a muy bajos dosados relativos o por el uso de altos niveles de gases recirculados (EGR). El alto nivel de dilución de la mezcla genera temperaturas de combustión promedio bajas y, como consecuencia, las emisiones de NOx son casi nulas; además, la mezcla es lo suficientemente homogénea como para disminuir la formación de MP. Debido a la ausencia de válvula mariposa y a las altas relaciones de compresión, los motores HCCI generan eficiencias térmicas comparadas a las obtenidas en MEC (Dec, 2009). Por otro lado, los motores HCCI presentan cantidades considerables de monóxido de carbono y combustible sin quemar en los gases de escape, esto último a causa de las bajas temperaturas de combustión que impiden que las reacciones de oxidación se completen y a la presencia de combustible en las zonas más frías de la cámara de combustión - ranuras, anillos, cabeza del pistón, entre otras (Dec, 2009; Hasan y Rahman, 2016; Saxena y Bedoya, 2013; Yao et al., 2009).

Aun cuando las ventajas de la combustión HCCI la hacen atractiva para los fabricantes de motores, existen algunos inconvenientes que deben ser solucionados antes de adoptarla en aplicaciones comerciales. Las investigaciones realizadas hasta la fecha han centrado sus esfuerzos en solucionar básicamente dos problemas:

\subsubsection{Falta de un sistema estandarizado de control de ignición}

La ignición es un fenómeno que depende de las características físicas y químicas de un sistema reactivo; en algunos equipos de uso industrial como quemadores, hornos y calderas, la ignición no representa un papel importante con relación al rendimiento, tipo de emisiones y otras características de operación del sistema; por el contrario, en MCIA y en especial en motores HCCI la ignición tiene gran interés (Westbrook, 2000). La ignición es uno de los factores más importantes que influencian las condiciones de funcionamiento de un motor HCCI, depende principalmente de la cinética química de la mezcla admitida, la cual a su vez está determinada por las condiciones de temperatura, presión, concentración y composición de los reactivos, además de otros parámetros geométricos y operacionales del motor como la relación de compresión (RC) y el régimen de giro (rpm) (Dec, 2009; Hasan y Rahman, 2016; Saxena y Bedoya, 2013; Yao et al., 2009). La interacción de todas estas variables ha dificultado el desarrollo de un método estandarizado de control de la ignición como en otras tecnologías, bujía en MEP e inyector en MEC. Una de las principales técnicas utilizadas para el control del inicio de la combustión es la variación de la temperatura de la mezcla admitida. Esta variación se puede obtener ya sea por calentamiento del aire de admisión, utilizando resistencias eléctricas, o por la variación en los tiempos del cierre de la válvula de escape (RCE), lo cual modifica la cantidad de gases residuales dentro de la cámara de combustión.

El momento en el que ocurre la ignición repercute en la potencia obtenida, la eficiencia térmica, la tasa de liberación de calor, la tasa de aumento de la presión, la transferencia de calor, la eficiencia de combustión, las emisiones contaminantes y los picos de temperatura y presión al interior del cilindro. Con una ignición adelantada, es decir, antes del punto muerto superior, se generan grandes pérdidas de potencia y eficiencia, altas tasas de aumento de la presión, aumento en las pérdidas de calor, alta producción de NOx y altas presiones al interior del cilindro. Por el contrario, con un excesivo retraso de la ignición, la potencia y eficiencia entregada por el motor disminuye a causa del poco trabajo aprovechado en la carrera de expansión; generando además una pobre eficiencia de combustión, lo que resulta en altas emisiones de HC y CO (Saxena y Bedoya, 2013). Para maximizar la cantidad de trabajo generado en un motor HCCI, es recomendado mantener el inicio de la combustión lo más cercano al punto muerto superior (PMS), algunos autores recomiendan que el CA50 - ángulo de giro del cigüeñal donde se ha libreado el $50 \%$ de la energía del combustible - se ubique entre 0 y $5{ }^{\circ} \mathrm{CA}$ antes del PMS (Hasan y Rahman, 2016; Saxena y Bedoya, 2013).

\subsubsection{Estrecho rango de operación}

En un motor HCCI estacionario la carga es controlada por la cantidad de combustible admitido o, en otras palabras, por el dosado relativo de la mezcla. Se entiende que a bajos requerimientos de carga los dosados relativos deben ser igualmente bajos, generando una baja eficiencia de 
combustión, lo que se refleja en altos niveles de combustible sin quemar y de monóxido de carbono en los gases de escape, debido a que a tan bajas cargas la mezcla está tan diluida que las temperaturas de combustión son muy bajas - 1500 $\mathrm{K}$ - y no es posible completar las reacciones de oxidación; también se puede alcanzar una alta variación de la presión media indicada o, incluso, ciclos de apagado (Dec, 2009; Hasan y Rahman, 2016). Por otro lado, los valores máximos de carga que puede tolerar un motor HCCI están limitados por las altas tasas de liberación de calor y de aumento de la presión, que de no controlarse pueden resultar en niveles de ruido intolerables al oído humano y en daños físicos del motor. Los motores HCCI de aspiración natural que funcionan con gasolina o diesel, solo alcanzan un tercio de la presión media indicada (PMI) que se obtiene en los motores convencionales de encendido provocado o encendido por compresión, alrededor de 5 bar (Saxena y Bedoya, 2013). Los valores típicos de dosado relativo reportados en la literatura para motores HCCI oscilan entre 0.20 y 0.50 (Hasan y Rahman, 2016), estos valores dependen del tipo de motor, tipo de combustible, temperatura y presión en la admisión y demás variables de operación del motor.

\subsection{Uso del gas de síntesis en motores HCCI}

Debido a las limitaciones causadas por el modo de combustión y la configuración del motor, las tecnologías de combustión tradicionales deben utilizar ciertos combustibles para garantizar su correcto funcionamiento. En MEP, gasolinas con alta volatilidad y gran resistencia a la autoignición son preferidas; mientras que, en MEC, diesel con buena viscosidad y baja resistencia a la autoignición es más apropiado. A diferencia de estos modos de combustión, un motor HCCI permite utilizar diferentes tipos de combustibles sin necesidad de alterar en mayor medida su configuración física. Ya que la ignición en un motor HCCI se debe a las condiciones cinéticoquímicas de la mezcla admitida, se hace necesario entender las características de ignición del combustible a utilizar, las cuales, como se comentó en párrafos anteriores, afectan de forma directa los parámetros de control y el rendimiento del motor. En términos generales, combustibles gaseosos o líquidos con alta volatilidad y que además presentan baja resistencia a la autoignición son los preferidos (Yao et al., 2009).

Pocos han sido los investigadores y los trabajos reportados en la literatura en los que se usa gas de síntesis como combustible en motores HCCI. En general, todos los trabajos buscan evaluar los parámetros de rendimiento y combustión del motor al variar la composición del gas de síntesis (Bhaduri et al, 2015; Haggith, 2011; Yamasaki et al, 2009). Ningún trabajo ha sido reportado en Colombia combinando motores HCCI y gas de síntesis. No se ven trabajos en condiciones de presión subatmosféricas, lo que abre un potencial de investigación de esta tecnología a estas condiciones.

\subsection{Modelo cero dimensional con cinética química detallada}

Los métodos de simulación numérica han resultado ser una gran herramienta para la investigación de la combustión HCCI puesto que son más versátiles y baratos que los métodos experimentales. Dado que la combustión HCCI depende en gran medida de la cinética química de la mezcla combustible-aire admitida, la cual a su vez depende de la composición, temperatura, presión y concentración de la mezcla; una buena aproximación del orden de magnitud de estas variables es indispensable para hacer un ajuste preliminar del sistema de control. El Modelo Cero Dimensional de una Zona con Cinética Química Detallada es el modelo para la simulación en motores más simplificado que existe, puesto que considera la temperatura, presión y concentración de la mezcla combustibleaire como homogénea en todo el volumen de la cámara de combustión y, el proceso de ignición y combustión se describe a partir de un mecanismo cinético detallado; aunque el modelo ha demostrado ser eficiente para predecir el momento de la ignición, presenta serias falencias al describir la tasa de liberación de calor, la duración de la combustión y las emisiones (Yao et al., 2009).

Desde el punto de vista de la cinética química, la ignición en un motor HCCI ocurre cuando el peróxido de hidrógeno (H2O2) que ha sido acumulado en la mezcla reactiva a partir de la reacción del hidroperóxido $(\mathrm{HO} 2)$, reacciones $(2)$ y (3), comienza a descomponerse a una temperatura cercana a los $1000 \mathrm{~K}$, reacción (4). Cuando la temperatura supera este valor se generan dos radicales hidroxilos $(\mathrm{OH})$ por cada $\mathrm{H} 2 \mathrm{O} 2$ que se descompone. Como resultado de la reacción anterior, la que hasta el momento era una molécula relativamente estable actúa como fuente de radicales $\mathrm{OH}$ los cuales consumen las moléculas de combustible más rápidamente, generando un aumento en la temperatura de los reactivos, ver Fig. 1. 


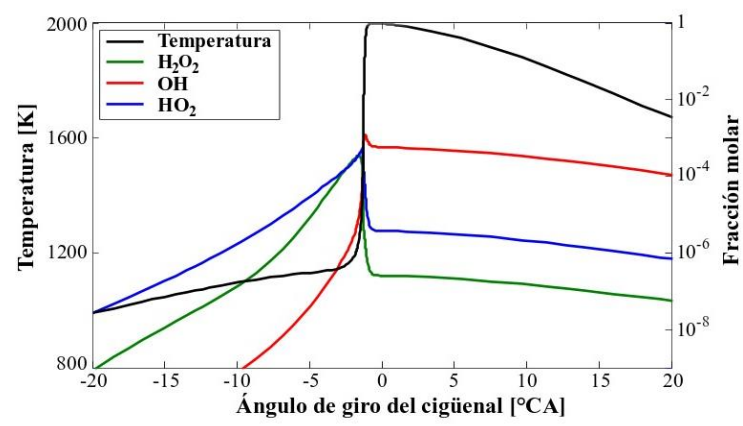

Fig. 1. Temperatura de la mezcla de reactivos y concentración de especies químicas $\left(\mathrm{H}_{2} \mathrm{O}_{2}, \mathrm{OH}\right.$ y HO2). Composición Cl, $\emptyset=0.30, T_{R C A}=475 \mathrm{Ky}$

$P_{R C A}=0.82$ bar. Fuente: elaboración propia.

Esta secuencia de reacciones continúa rápidamente hasta que la temperatura de la mezcla alcanza los $1200 \mathrm{~K}$, temperatura en la cual la reacción de ramificación (1) toma lugar y domina el resto de la reacción global (Warnatz et al, 1996; Westbrook, 2000). Cualquier parámetro de funcionamiento del motor que acelere el momento de descomposición del $\mathrm{H} 2 \mathrm{O} 2$, es causante de un adelanto de la autoignición de la mezcla reactiva.

$$
\begin{aligned}
& \mathrm{H}+\mathrm{O}_{2} \rightarrow \mathrm{O}+\mathrm{OH} \\
& \mathrm{H}+\mathrm{O}_{2}+\mathrm{M} \rightarrow \mathrm{HO}_{2}+\mathrm{M} \\
& \mathrm{RH}+\mathrm{HO}_{2} \rightarrow \mathrm{R}+\mathrm{H}_{2} \mathrm{O}_{2} \\
& \mathrm{H}_{2} \mathrm{O}_{2}+\mathrm{M} \rightarrow \mathrm{OH}+\mathrm{OH}+\mathrm{M}
\end{aligned}
$$

Donde $\mathrm{RH}$ es un alcano, $\mathrm{R}$ es un radical alquilo, $\mathrm{y}$ $\mathrm{M}$ es un tercer cuerpo.

En este estudio, se analizan los efectos de la variación en la composición, el dosado relativo y las condiciones de temperatura y presión en la admisión como parámetros de control de la autoignición del gas de síntesis en un motor estacionario para microgeneración funcionando en modo HCCI; a través de un análisis numérico utilizando un modelo cero dimensional de una zona con cinética química detallada, empleando el programa CHEMKIN-PRO®.

\section{METODOLOGÍA}

Los parámetros geométricos utilizados en el estudio corresponden a los de un motor diesel Lombardini 25 LD 425-2 refrigerado por aire, el cual ha sido modificado para trabajar en modo HCCI. Dentro de las modificaciones realizadas se incluye el cambio de la cabeza del pistón por una de geometría plana y el cambio del régimen de giro nominal. La Tabla 1 resume los principales parámetros geométricos que alimentan el modelo computacional.

\section{Tabla 1: Parámetros geométricos del motor}

\begin{tabular}{|c|c|c|}
\hline Parámetro & Valor & Unidad \\
\hline Cilindrada & 851 & $\mathrm{~cm} 3$ \\
\hline Numero de cilindros & 2 & - \\
\hline Diametro del cilindro & 8.5 & $\mathrm{~cm}$ \\
\hline Relación de compresión & 17.635 & - \\
\hline Regimen de giro & 1800 & $\mathrm{rpm}$ \\
\hline Longitud de la biela & 11.75 & $\mathrm{~cm}$ \\
\hline Radio del cigüeñal & 3.75 & $\mathrm{~cm}$ \\
\hline $\begin{array}{c}\text { Periodo de valvulas } \\
\text { cerradas }\end{array}$ & 210 & ${ }^{\circ} \mathrm{CA}$ \\
\hline $\begin{array}{c}\text { Cierre de la valvula de } \\
\text { admisión (aRCA) }\end{array}$ & -115 & ${ }^{\circ} \mathrm{CA}$ \\
\hline
\end{tabular}

Fuente: elaboración propia.

Se utiliza el mecanismo cinético detallado San Diego, desarrollado por la UC San Diego para investigación en llamas, ignición y detonación. Se evalúan tres composiciones diferentes del gas de síntesis - C1, C2 y C3 -, consideradas a partir de los valores típicos obtenidos en gasificadores de lecho fijo que utilizan aire como agente gasificante (McKendry, 2002c). En cada una de las composiciones evaluadas se mantiene contante los porcentajes volumétricos de metano, dióxido de carbono y nitrógeno. Solo se considera la variación en los porcentajes de hidrógeno y oxígeno. Las composiciones de gas de síntesis y algunas propiedades de combustión relevantes se muestran en la Tabla 2.

\section{Tabla 2: Composiciones del gas de síntesis y propiedades de combustión}

\begin{tabular}{|c|c|c|c|}
\hline \multirow{2}{*}{ Variable } & \multicolumn{3}{|c|}{ Composición } \\
\cline { 2 - 4 } & C1 & C2 & C3 \\
\hline $\mathrm{H}_{2}[\%$ Vol. $]$ & 20 & 15 & 10 \\
\hline $\mathrm{CO}[\%$ Vol.] & 15 & 20 & 25 \\
\hline $\mathrm{CH}_{4}[\%$ Vol. $]$ & \multicolumn{3}{|c|}{5} \\
\hline $\mathrm{CO}_{2}[\%$ Vol. $]$ & \multicolumn{3}{|c}{50} \\
\hline $\mathrm{N}_{2}[\%$ Vol. $]$ & \multicolumn{3}{|c|}{} \\
\hline $\mathrm{H}_{2} / \mathrm{CO}[-]$ & 1.33 & 0.75 & 0.40 \\
\hline $\mathrm{PCI}[\mathrm{kJ} / \mathrm{kg}]$ & 5498.5 & 5295.8 & 5113.0 \\
\hline $\mathrm{PCI}\left[\mathrm{kJ} / \mathrm{Nm}^{3}\right]$ & 5443.5 & 5528.9 & 5614.4 \\
\hline AFRs $\left[\mathrm{kg}_{\text {aire }} / \mathrm{kg}_{\text {combustible }}\right]$ & 1.59 & 1.51 & 1.44 \\
\hline
\end{tabular}

Fuente: elaboración propia.

El estudio se realiza tomando la temperatura en el RCA como variable paramétrica. Se considera la transferencia de calor en la mezcla reactiva desde y hacia las paredes; el modelo computacional la determina a partir de la correlación semiempírica 
de Woschni [Woschni]. La temperatura de la pared es función del dosado relativo y se calcula a partir de ecuación (1).

$$
T_{\text {pared }}=79.258 * \emptyset+402.15
$$

Las variables de operación del motor y los valores que se consideran en el estudio se muestran en la Tabla 3.

\section{Tabla 3: Parámetros para simulación}

\begin{tabular}{|c|c|c|}
\hline Variable & Valor & Unidad \\
\hline $\begin{array}{c}\text { Temperatura en } \\
\text { el RCA }\end{array}$ & Paramétrica & $\mathrm{K}$ \\
\hline Presión en el & 0.82 & bar \\
RCA & 0.90 & \\
& 1.00 & \\
& 0.20 & \\
Dosado relativo & 0.25 & - \\
(ळ) & 0.30 & \\
& 0.35 & \\
\hline Temperatura de & 0.45 & $\mathrm{~K}$ \\
la pared & 0.50 & $T(\emptyset)$ \\
\hline
\end{tabular}

Fuente: elaboración propia.

\section{RESULTADOS}

En la Fig. 2 se muestra el mapa de ignición de la composición $\mathrm{C} 1$ en función de la temperatura en el RCA para todos los valores de dosado relativo y una presión en el RCA de 0.82 bar. Como se comentó en la sesión introductoria, tanto la temperatura y presión en el RCA como el dosado relativo tienen un efecto significativo en el momento de la autoignición. Y es que, las tasas de reacción son altamente sensibles a la temperatura, presión y concentración de la mezcla de reactivos (McAllister et al, 2011). Para un valor constante de dosado relativo un incremento en la temperatura de admisión genera un adelanto en el ángulo de ignición; este efecto es ligeramente mayor en el rango de temperaturas que generan ignición antes del PMS. Para una temperatura en el RCA constante, un aumento en el dosado relativo produce un adelanto en el momento de la ignición; la variación en el ángulo de ignición es menor conforme la mezcla admitida se torna más rica.

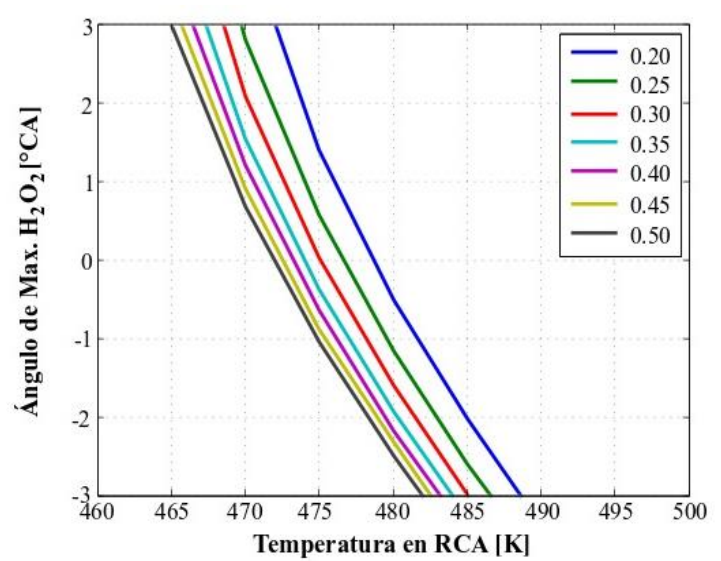

Fig. 2. Mapa de ignición en función de la TRCA para todos los valores de dosado relativo. Composición C1 y PRCA=0.82 bar. Fuente: elaboración propia.

El efecto de la variación de la composición del gas de síntesis para una presión en el RCA constante en función de la temperatura en el RCA para todos los valores de dosado relativo se puede ver en la Fig. 3, cuadros (a) y (b). En general, los mapas de ignición sufren un desplazamiento a la derecha conforme la cantidad de $\mathrm{H} 2$ en la mezcla disminuye y la de CO aumenta; lo que equivale a decir que para un mismo dosado relativo y ángulo de ignición, los requerimientos de temperatura en el RCA son menores conforme la cantidad de hidrógeno en la mezcla es mayor. Esto se debe a que la reacción entre el $\mathrm{CO}$ y el $\mathrm{O} 2$ es un proceso muy lento, incluso a altas temperaturas, reacción (R.5), mientras que, pequeñas cantidades de $\mathrm{H} 2$ en la mezcla tienen el efecto de acelerar las tasas de reacción global, reacción (R.6) (Sung y Law, 2008).

$\mathrm{CO}+\mathrm{O}_{2} \rightarrow \mathrm{CO}_{2}+\mathrm{O}$

$\mathrm{CO}+\mathrm{HO}_{2} \rightarrow \mathrm{CO}_{2}+\mathrm{OH}$

En la Fig. 3, cuadros (c) y (d), se evalúa el efecto de la variación de la presión en el RCA para la composición $\mathrm{C} 1$ en función de la temperatura en el RCA y para todos los valores de dosado relativo. Para un mismo dosado relativo y temperatura en el RCA, un aumento de la presión en el RCA causa un adelanto del inicio de la combustión o menores temperaturas de admisión son requeridas si se quiere mantener el ángulo de ignición. Sin embargo, el efecto de la variación de la presión en el RCA es inferior al de la variación de la composición y al de la temperatura, por lo menos en los niveles evaluados en el presente trabajo. 


\section{CONCLUSIONES}

El ángulo de ignición repercute en la eficiencia, potencia, tasa de liberación de calor, picos máximos de presión, estabilidad de la combustión, emisiones y demás parámetros de operación, combustión y rendimiento de un motor HCCI. La ignición es controlada por la composición, concentración, temperatura y presión de la mezcla de reactivos admitida. En este trabajo se evaluó la variación de las variables anteriormente nombradas en la autoignición del gas de síntesis haciendo uso de un modelo computacional cero dimensional de una zona con cinética química detallada. Las principales conclusiones son:

1. Existe una alta dependencia de la ignición con la temperatura en el RCA. Los valores de temperatura que generan centrado en la

(a)

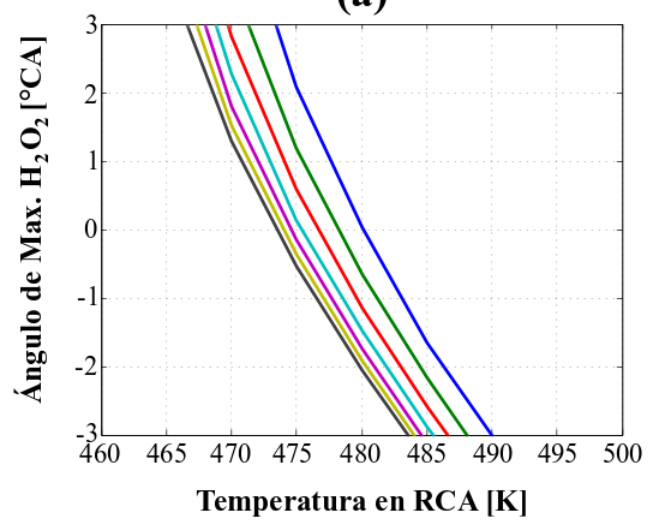

(c)

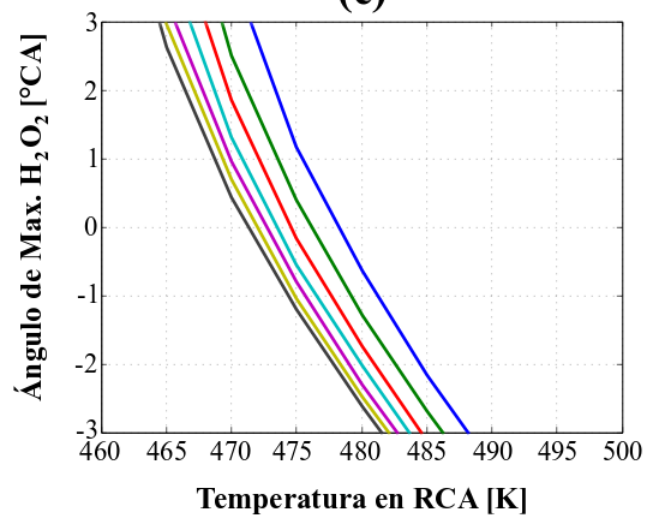

ignición para todos los puntos evaluados están comprendidos entre $465 \mathrm{~K}$ y $495 \mathrm{~K}$.

2. Para una composición y temperatura constantes un aumento de la concentración del combustible adelanta el inicio de la combustión, este efecto se torna menor conforme el dosado relativo se hace más grande.

3. Para una temperatura en el RCA y dosado relativo constantes el incremento en la cantidad de $\mathrm{H} 2$ en el combustible adelanta la ignición $\mathrm{o}$ menores temperaturas son requeridas si se quiere mantener el ángulo de ignición.

4. El efecto de la presión en el RCA es similar pero de menor magnitud al de la variación de la composición y al de la temperatura de admisión, por lo menos en los niveles evaluados en el presente trabajo. (b)

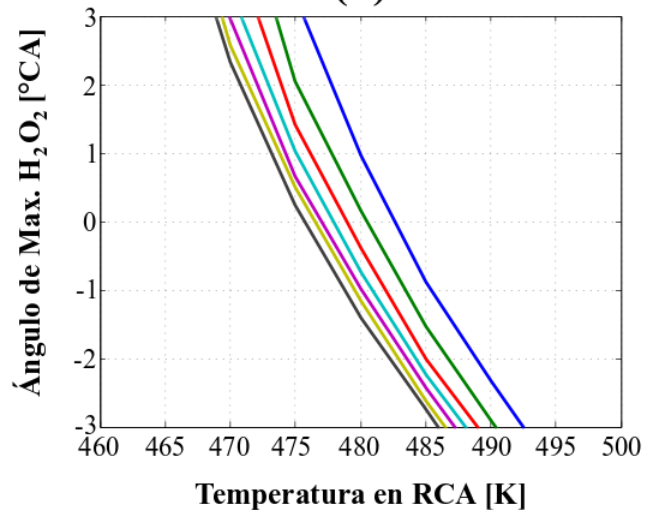

(d)

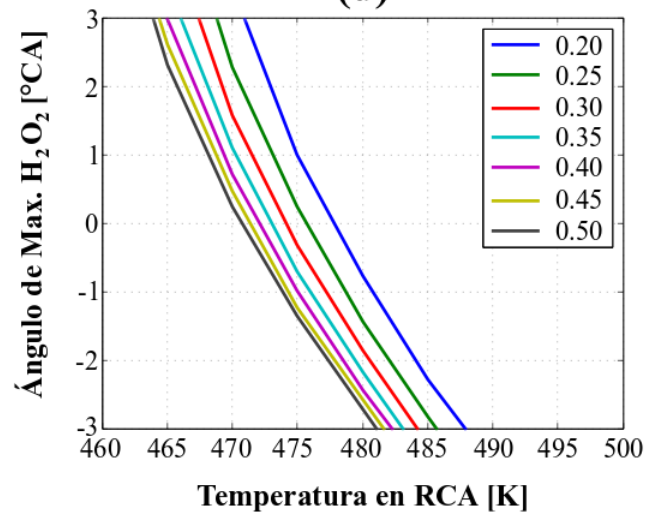

Fig. 3. Mapa de ignición en función de la $T_{R C A}$ para todos los valores de dosado relativo. Cuadros (a) y (b) composición C2 y C3, respectivamente y $P_{R C A}=0.82$ bar. Cuadros (c) y (d) composición Cl y $P_{R C A}$ de 0.90 y 1.00 bar, respectivamente. Fuente: elaboración propia. 


\section{AGRADECIMIENTOS}

Al Grupo de Investigación del Gas y Uso Racional de la Energía (GASURE) de la Universidad de Antioquía, al programa Estimulo Académico de Estudiante Instructor de la Universidad de Antioquia y a la Institución Universitaria Pascual Bravo.

\section{REFERENCIAS}

Agency, I. E. (2018). Key world energy statistics. International Energy Agency Paris.

AB Niño (2018) Micro turbina Peltón, una solución real de energía para zonas no interconectadas (ZNI). Revista Colombiana de Tecnologías de Avanzada, ISSN: 1692-7257

Bhaduri, S., Contino, F., Jeanmart, H., y Breuer, E. (2015). The effects of biomass syngas composition, moisture, tar loading and operating conditions on the combustion of a tar-tolerant HCCI (Homogeneous Charge Compression Ignition) engine. Energy, 87, 289-302. https://doi.org/10.1016/j.energy.2015.04.076

Ciferno, J. P., y Marano, J. J. (2002). Benchmarking biomass gasification technologies for fuels, chemicals and hydrogen production. In US Department of Energy. National Energy. Retrieved from http://seca.doe.gov/technologies/coalpower/ga sification/pubs/pdf/BMassGasFinal.pdf

Dec, J. E. (2009). Advanced compression-ignition engines - understanding the in-cylinder processes. Proceedings of the Combustion Institute, 32, 2727-2742. https://doi.org/10.1016/j.proci.2008.08.008

Escalante, H., Orduz, J., Zapata, H., Cardona, M. C., y Duarte, M. (2011). Atlas del potencial energético de la biomasa residual en Colombia. Anexo B: Muestreo y Caracterización de La Biomasa Residual En Colombia (Págs. 131-136). Colombia.

Esteve Gómez, N. (2011). Energización de las zonas no interconectadas a partir de las energías renovables solar y eólica. Facultad de Estudios Ambientales y Rurales.

Haggith, D. E. (2011). Combustion Phasing and Engine Performance of an HCCI Engine Utilizing Simulated Biomass Gas. University of Windsor.

Hagos, F. Y., Aziz, A. R. A., y Sulaiman, S. A. (2014). Trends of syngas as a fuel in internal combustion engines. Advances in Mechanical Engineering, 6, 401587.
Hasan, M. M., y Rahman, M. M. (2016). Homogeneous charge compression ignition combustion: Advantages over compression ignition combustion, challenges and solutions. Renewable and Sustainable Energy Reviews, 57, 282-291. https://doi.org/10.1016/j.rser.2015.12.157

Heywood, J. B. (1988). Internal Combustion Engine Fundementals. In McGrawHill series in mechanical engineering (Vol. 21). https://doi.org/10987654.

JEG Plaza, MAR Nuñez, (2017) Formación en competencias específicas para la industria del software colombiano. Experiencias del uso del aprendizaje basado en proyectos. Revista Colombiana de Tecnologías de Avanzada, ISSN: 1692-7257.

L Tangarife, M Sánchez, M Rojas (2017). Modelo de interventoría de tecnologías de información en el área de conocimiento de la gestión del alcance de PMBOK® y alineado con ISO 21500 y COBIT®. Revista Colombiana de Tecnologías de Avanzada, ISSN: 1692-7257

McAllister, S., Chen, J.-Y., y Fernandez-Pello, A. C. (2011). Fundamentals of combustion processes (Vol. 302). Springer.

McKendry, P. (2002a). Energy production from biomass (part 1): overview of biomass. Bioresource Technol, 83(1), 37-46. https://doi.org/10.1016/S09608524(01)00118-3

McKendry, P. (2002b). Energy production from biomass (part 2): Conversion technologies. Bioresource Technology, 83(1), 47-54. https://doi.org/10.1016/S09608524(01)00119-5

McKendry, P. (2002c). Energy production from biomass (part 3): Gasification technologies. Bioresource Technology, 83(1), 55-63. https://doi.org/10.1016/S09608524(01)00120-1

Quaak, P., Harrie, K., y Stassen, H. (1999). Energy from biomass: a review of combustion and gasification technologies (Vol. 23). World Bank Publications.

O Suarez, C Vega, E Sánchez, A Pardo. (2018) Degradación anormal de p53 e inducción de apoptosis en la red P53-mdm2 usando la estrategia de control tipo pin. Revista Colombiana de Tecnologías de Avanzada, ISSN: 1692-7257.

Rey Luengas, O. L., Vélez, O. F., Serrano, S., Zabaleta, O. A., y Sáenz, G. (2018). Zonas no interconectadas -ZNI Diagnóstico de la prestación del servicio de energía eléctrica. Bogotá D.C. 
Richards, G. A., Casleton, K. H., y Weiland, N. T. (2009). Syngas utilization. Synthesis Gas Combustion Fundamentals and Application, 197-222.

Saxena, S., y Bedoya, I. D. (2013). Fundamental phenomena affecting low temperature combustion and HCCI engines, high load limits and strategies for extending these limits. Progress in Energy and Combustion Science, 39, 457-488. https://doi.org/10.1016/j.pecs.2013.05.002 Review

Sung, C.-J., y Law, C. K. (2008). Fundamental Combustion Properties of H2/CO Mixtures: Ignition and Flame Propagation at Elevated Pressures. Combustion Science and Technology, 180(6), 1097-1116. https://doi.org/10.1080/00102200801963169

Warnatz, J., Maas, U., Dibble, R. W., y Warnatz, J. (1996). Combustion (Vol. 3). Springer.

Westbrook, C. K. (2000). Chemical kinetics of hydrocarbon ignition in practical combustion systems. Proceedings of the Combustion Institute, 28(2), 1563-1577.

Yamasaki, Y., Kanno, M., Taura, Y., y Kaneko, S. (2009). Study on Biomass Gas HCCI Engine. SAE Technical Paper, 32(0066), 7.

Yao, M., Zheng, Z., y Liu, H. (2009). Progress and recent trends in homogeneous charge compression ignition (HCCI) engines. Progress in Energy and Combustion Science, 35 , 398-437. https://doi.org/10.1016/j.pecs.2009.05.001

\section{SITIOS WEB}

Emission Standards. (n.d.). Retrieved from https://www.dieselnet.com/standards/

"Chemical-Kinetic Mechanisms for Combustion Applications", San Diego Mechanism web page,

Mechanical and Aerospace Engineering (Combustion Research), University of California at San Diego (http://combustion.ucsd.edu).ion institute, 28(2), 1563-1577. 